\title{
Modelling the impact of extended vaccination strategies on the epidemiology of pertussis
}

\author{
M. H. ROZENBAUM*, R. De VRIES, H. H. LE And M. J. POSTMA \\ Unit of PharmacoEpidemiology and PharmacoEconomics $\left(P E^{2}\right)$, Department of Pharmacy, University of \\ Groningen, Groningen, The Netherlands
}

(Accepted 30 October 2011; first published online 24 November 2011)

\section{SUMMARY}

The aim of this study was to investigate the optimal pertussis booster vaccination strategy for The Netherlands. A realistic age-structured deterministic model was designed. Assuming a steady-state situation and correcting for underreporting, the model was calibrated using notification data from the period 1996-2000. Several sensitivity analyses were performed to explore the impact of different assumptions for parameters surrounded by uncertainty (e.g. duration of protection after natural infection, underreporting factors, and transmission probabilities). The optimal age of an additional booster dose is in the range of 10-15 years, and implementation of this booster dose will reduce both symptomatic and asymptomatic infections, although the incidence of symptomatic infections in older age groups will increase. The impact of the different assumptions used in the model was in general limited. We conclude that over a wide range of assumptions, an additional booster dose can reduce the incidence of pertussis in the population.

Key words: Infectious disease control, mathematical modelling, pertussis (whooping cough), public health, vaccine policy development.

\section{INTRODUCTION}

Bordetella pertussis is a bacterium that causes the highly contagious respiratory disease pertussis, also known as whooping cough. Despite widespread vaccination, infection with pertussis remains endemic even in countries with high vaccination coverage [1-4]. Moreover, there has been a resurgence of pertussis in many countries during the past decade, particularly in adolescents and adults [1, 3, 5-7]. For example, in The Netherlands a clear increase in the incidence of pertussis was apparent from 1996

\footnotetext{
* Author for correspondence: Dr M. H. Rozenbaum, University of Groningen, Antonius Deusinglaan 1, 9713 AV Groningen, The Netherlands.

(Email: m.h.rozenbaum@rug.nl)
}

onwards despite a consistently high vaccine uptake $[4,5]$. Although infections in adolescents and adults are less severe than those in infants and young children, the increasing incidence in adolescents and adults is still a major concern because adolescents and adults are identified as important sources of transmission to young infants who are not yet vaccinated or only partially vaccinated [8-10]. Therefore, the addition of immunization strategies to the current childhood immunization programme should be considered not only to reduce the disease burden in adolescents and adults but also to prevent transmission of the infection to infants. Indeed, several countries (e.g. Australia, France, USA) have already incorporated adolescent booster doses into their national immunization programmes [11, 12]. However, other

The online version of this article is published within an Open Access environment subject to the conditions of the Creative Commons Attribution-NonCommercial-ShareAlike licence $<$ http://creativecommons.org/licenses/by-nc-sa/2.5/ $>$. The written permission of Cambridge University Press must be obtained for commercial re-use. 


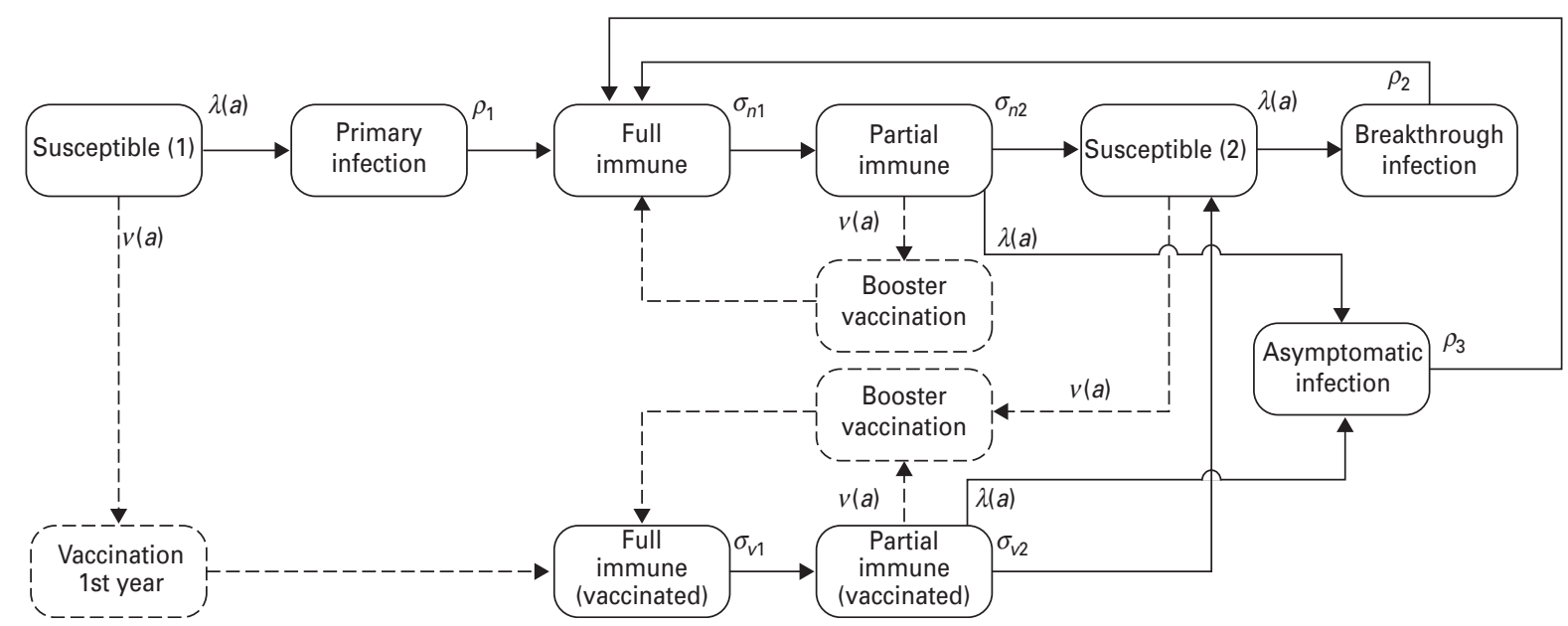

Fig. 1. Graphical representation of the possible pathways within the model. The solid compartments represent the different pertussis epidemiological states. Solid arrows represent the flow between these states. Dashed lines and compartments represent events and pathways associated with vaccination.

countries such as The Netherlands, have not implemented adolescent or adult booster immunization programmes even though such programmes would potentially reduce the transmission of $B$. pertussis and consequently lower the incidence of pertussis in infants $[10,13,14]$. Therefore, the impact of additional booster dose(s) at later age(s) should be explored.

Mathematical models can be used to investigate, for example, the optimal age(s) of vaccination or the impact of different levels of uptake of the vaccine. During the last 10 years several studies have modelled the potential impact of additional booster doses [14-19]. Nevertheless, specific drawbacks make them inappropriate to use for decision making. For example, most studies did not (i) take into account underreporting (for adults), (ii) explore the impact on the epidemiological outcome of transmission-related parameters, or (iii) use contact rates based on 'real life' contact patterns. Also, our previous work had these limitations [17]. We have attempted to overcome these limitations through the use of a realistic agestructured deterministic model, programmed within an environment allowing for high-speed model runs, to determine the optimal vaccination strategy for reducing the number of infections in the population, and to explore the impact of different assumptions for parameters surrounded by uncertainty.

\section{MATERIALS AND METHODS}

Several analyses were performed to explore the impact of introducing different types of booster immunization programmes to the current Dutch vaccination strategy in which acellular vaccine formulations are used and infants receive three doses at the ages of 2, 3 and 4 months and booster doses at 11 months and 4 years. In particular, we focused on the optimal age of implementing a single additional booster vaccination. However, we also investigated other potential vaccination strategies to reduce the burden of disease in the youngest age groups such as providing a booster dose every 10 years. Base-case, sensitivity and scenario analyses were performed. The base-case analysis represented the most plausible assumptions (described below) and was subsequently used as a baseline against which all other scenarios and sensitivity analyses were assessed. The dynamic model was programmed in Berkeley Madonna (R. I. Macey \& G. F. Oster, UC Berkeley, CA, USA).

\section{Model structure}

A schematic overview of the deterministic model used to assess the impact of various booster vaccination strategies against pertussis is shown in Figure 1, where mutually exclusive compartments represent the different epidemiological states of the disease and the arrows represent possible flows of individuals between the different states. Note that the underlying structure of the model is the same as our previously published stochastic model [17]. In the model all infants are born susceptible and then face age-specific risks of acquiring a pertussis infection as a result of contact with an infectious person. As shown in Figure 1, the model distinguished between three types of infections: (i) infections in immunologically naive individuals 
Table 1. Epidemiological data

\begin{tabular}{|c|c|c|}
\hline Variable & $\begin{array}{l}\text { Parameter } \\
\text { (see also Fig. 1) }\end{array}$ & Base-case value \\
\hline Annual birth cohort & n.a. & $100000 / 75$ \\
\hline Force of infection & $\lambda(a)$ & $\begin{array}{l}\text { Age-dependent } \\
\text { (see Supplementary Fig. S1) }\end{array}$ \\
\hline Rate of recovery from primary infection & $\rho_{1}$ & $13 \cdot 0\left(\mathrm{yr}^{-1}\right)$ \\
\hline $\begin{array}{l}\text { Rate of recovery from breakthrough } \\
\text { infection }\end{array}$ & $\rho_{2}$ & $17 \cdot 4\left(\mathrm{yr}^{-1}\right)$ \\
\hline Rate of recovery from asymptomatic infection & $\rho_{3}$ & $52 \cdot 1\left(\mathrm{yr}^{-1}\right)$ \\
\hline Rate of loss of full immunity after primary infection & $\sigma_{n 1}$ & $0 \cdot 50\left(\mathrm{yr}^{-1}\right)$ \\
\hline Rate of loss of partial immunity after vaccination & $\sigma_{n 2}$ & $0 \cdot 17\left(\mathrm{yr}^{-1}\right)$ \\
\hline Rate of loss of full immunity after vaccination & $\sigma_{v 1}$ & $0 \cdot 50\left(\mathrm{yr}^{-1}\right)$ \\
\hline $\begin{array}{l}\text { Rate of loss of partial immunity after breakthrough } \\
\text { infection }\end{array}$ & $\sigma_{v 2}$ & $0 \cdot 10\left(\mathrm{yr}^{-1}\right)$ \\
\hline Fraction effectively protected by vaccination & $V(a)^{*}$ & Age dependent \\
\hline Vaccination coverage first 4 doses & n.a. & $0 \cdot 96$ \\
\hline Vaccination coverage booster at age 12 years & n.a. & $0 \cdot 7$ \\
\hline Vaccine efficacy $\dagger$ & n.a. & $0 \cdot 89$ \\
\hline
\end{tabular}

* $(a)=$ age.

$\dagger$ We assumed that a certain fraction of the population (coverage $\times$ efficacy) is effectively protected precisely after 4 months for vaccinated infants and after 4 years for the first booster dose. The age of protection after the third booster dose was dependent on the age at which this dose was administered.

(henceforth called primary infections); (ii) infections in individuals whose immune system has been primed by vaccination or infection (breakthrough infections, sometimes known as 'recidive' infections); and (iii) asymptomatic infections (note that all primary and breakthrough infections were assumed to be symptomatic).

Individuals were assumed to be fully immune (i.e. immunity against transmission and disease) to subsequent infections following either vaccination or recovery from primary infection. Because of waning of immunity, these individuals will become partially immune (i.e. immunity against disease only). Partially immune individuals can acquire and transmit the pathogen but will not become ill and only experience asymptomatic infections. However, partial immunity also wanes with time. As a result, partially immune individuals will again become susceptible. These susceptible individuals are at risk of acquiring breakthrough infections, which were assumed to be less severe than primary infections because the immune system had previously been primed. Furthermore, we assumed that partially immune and susceptible individuals can re-acquire full immunity as a result of contact with the pathogen through either vaccination or subsequent infection.

\section{Disease characteristics}

Although the duration of the infectious period is not precisely known and is likely to vary between individuals, it has been suggested to be dependent on the severity of the disease [17]. Based on expert estimations, we assumed the average infectious period for individuals with primary infections, breakthrough infections, and asymptomatic infections to be 4 weeks, 3 weeks, and 1 week, respectively (see Table 1 for specific parameter values used) [17].

Similar to the duration of the infectious period, the duration of immunity after a natural infection is not precisely known. However, a recent review suggested that immunity after a natural infection wanes after 4-20 years [20]. Based on these data, we assumed in the baseline analysis that immunity after natural infection wanes after 12 years on average, with individuals being fully protected for 2 years and partially protected for 10 years.

\section{Vaccine characteristics and vaccination schedules}

Until 2001, the vaccination schedule in The Netherlands consisted of four doses (currently at ages 2, 3, 4 months and a booster at age 11 months) of the whole cell pertussis vaccine within the combination of 
diphtheria, tetanus and polio vaccine. From 2001 onwards, an additional acellular booster vaccination was given to 4-year-old children. Starting in 2005 the pertussis component in the combination vaccine was changed from the whole cell to an acellular vaccine. The efficacy of the whole cell pertussis vaccine was estimated at $89 \%[4,21-23]$. Based on clinical trial data, we also assumed that the vaccine efficacy after vaccination in the first year [24, 25], after booster vaccination at age 4 years [24, 25], and after adolescent booster vaccination would be $89 \%$ [26-28].

In the model the vaccination scheme was divided into three parts: (i) the vaccinations administrated in the first year (including the first booster dose at age 11 months); (ii) a second booster dose at age 4 years; and (iii) potentially a third booster dose at age 12 years. After vaccination, we assumed that the fraction of the population, defined by coverage multiplied by efficacy, is effectively protected precisely after 4 months. Using a recent estimate for duration of immunity after vaccination with either whole cell or acellular vaccine of 4-12 years [20], we assumed that the immunity acquired by vaccination would be for 8 years, where individuals were fully and partially protected for 2 and 6 years, respectively. Note, that the duration of full immunity after vaccination is identical to the duration after natural infection, while the duration of partial immunity is 4 years shorter after vaccination compared to natural infection.

Based on the actual pertussis vaccination coverage of the Dutch national immunization programme, we applied a vaccine uptake of $96 \%$ for the three infant doses and the two booster doses (at ages 11 months and 4 years). Vaccine coverage of $70 \%$ was assumed for the adolescent booster dose(s), which is much lower than the coverage for infants but is still higher than the uptake achieved in girls with the more controversial human papillomavirus vaccine in The Netherlands $(\sim 50 \%)$. In the sensitivity and scenario analyses the vaccination coverage was varied over a much wider range.

\section{Force of infection (FOI)}

The FOI is the rate at which susceptible individuals will be infected within a given time period. Agedependent FOIs were estimated by using a method developed by van Boven et al. [21] (see also Supplementary online material). The main advantage of this method is that it is able to take different types of infection and waning immunity into account in a consistent manner [21].

Because an additional booster dose at age 4 years was introduced in 2001, we assumed an endemic equilibrium from 1996 to 2000. For this period, average age-specific incidences were calculated based on case notification data after correction for underreporting $[29,30]$. Previously, it was estimated that the incidence of pertussis including very mild and asymptomatic cases in The Netherlands was more than 600 times higher than the notified cases for children and adults [29]. In particular, these age-specific Dutch ratios of underreporting were used to correct the number of notified cases [29]. We note that in reality there was probably no endemic equilibrium from 1996 to 2000 [30]. Once the FOIs were estimated these were subsequently used to calculate age-specific transmission coefficients. The transmission coefficient denotes the probability that a contact between a susceptible individual of a specific age and an infectious individual of a specific age leads to transmission. Since the transmission coefficients $(\beta)$ can be expressed as a function of the number of infectious individuals at a given point in time, the contact function and FOIs, the transmission coefficients can be calculated once the age-specific FOIs are known. The contact function represents the number of contacts between an individual in one specific age group with an individual in another age group per unit of time. We applied the contact function for respiratory diseases in the Dutch population estimated by Wallinga et al. [31]. We assumed that the transmission probability would increase with severity of disease and assigned transmission probabilities of $1,0 \cdot 7$, and $0 \cdot 05$ for primary $\left(\beta_{\text {primary }}\right)$, breakthrough $\left(\beta_{\text {breakthrough }}\right)$ and asymptomatic ( $\left.\beta_{\text {asymptomatic }}\right)$ infection, respectively.

\section{Population}

Simulations were performed for a population of 100000 individuals with a uniform age distribution (i.e. the age groups were equally sized). The population was divided into 86 age groups, represented by 1 -month groups for the first year $(0-11$ months) and 1-year groups subsequently (1-74 years). The total population size remained constant because newborns entering the model were equal to individuals leaving the model from death (at the age of 75). To mimic reality, the model was started at the steady state of the 
Table 2 a. Sensitivity analyses performed on the base-case analysis

\begin{tabular}{|c|c|c|}
\hline & Base case & Sensitivity analysis \\
\hline Variation in the coverage of the booster dose & $70 \%$ & $50-90 \%(10 \%)$ \\
\hline Contact function & Based on Wallinga et al. [31] & Based on Mossong et al. [32] \\
\hline $\begin{array}{l}\text { Different transmission probabilities } \\
\beta_{\text {primary }}(a): \beta_{\text {breakthrough }}(a): \beta_{\text {asymptomatic }}(a)\end{array}$ & $1: 0 \cdot 7: 0 \cdot 05$ & $\begin{array}{l}1: 1: 0 \cdot 05 \\
1: 0 \cdot 7: 0 \\
1: 0 \cdot 7: 0 \cdot 10\end{array}$ \\
\hline Duration of protection after natural infection & $12(2+10)$ in years & $\begin{array}{l}8(2+6) \text { in years } \\
16(2+14) \text { in years }\end{array}$ \\
\hline $\begin{array}{l}\text { Duration of infectiousness for primary, } \\
\text { breakthrough and asymptomatic infections (in weeks) }\end{array}$ & $4,3,1$ & $\begin{array}{l}2,2,1 \\
3,3,1\end{array}$ \\
\hline Reducing the estimated underreporting factors & $0 \%$ & $\begin{array}{l}25 \% \\
50 \%\end{array}$ \\
\hline
\end{tabular}

Table $2 b$. Scenarios (variations on base-case analysis) investigated and sensitivity analyses

\begin{tabular}{ll}
\hline \hline Scenarios & Age booster(s) (in years) \\
\hline Current situation (without adolescent booster dose) & n.a. \\
Base case & 12 years \\
Age of the first booster dose (adolescent vaccination) & Between 5 and 35 years $\dagger$ \\
Combined adolescent (1 dose) and adult immunization & Third booster dose between 10 and 18 years and \\
$(1$ dose) & fourth booster dose between 18 and 35 years $\dagger$ \\
A booster dose every 10 years starting at age 10 years until & $10,20,30,40,50,60,70 \dagger$ \\
age 60 years & \\
\hline \hline
\end{tabular}

* All possible combinations were investigated (applying a minimal period between the doses of at least 5 years, and assuming the youngest age at which an adolescent booster is administered as 10 years).

$\dagger$ A step size of 1 year was used.

1996-2000 endemic period $(t=-5$ to -1$)$ with a booster vaccination at age 4 years implemented in $2001(t=0)$. Additionally, we assumed in the basecase analysis that a booster dose at age 12 years would be implemented in $2011 \quad(t=10)$ and the impact of this booster vaccination on pertussis incidence and prevalence in the population was assessed over a time period of 35 years. All vaccinations were assumed to be administered at the start of a new year.

\section{Sensitivity analysis and scenario analysis}

In the base-case scenario we used the most plausible parameter assumptions. However, as many parameters are surrounded by some level of uncertainty, we performed several sensitivity analyses to explore the impact of this uncertainty on the epidemiological outcome (Table $2 a$ ). To explore the impact of vaccination coverage of the booster dose, coverage was varied over a range of $50-90 \%$ by increments of $10 \%$. In addition, the impact of applying an alternative contact function based on data provided by Mossong et al. [32] was explored. The advantage of using the data of Mossong et al. is that it is more recent; however a disadvantage is the much smaller sample size compared to the data of Wallinga et al. [31]. Furthermore, the transmission probabilities of the different types of infections, the duration of protection after natural immunity, and the duration of infectiousness were varied [20]. Finally, as the real incidence of pertussis is surrounded by uncertainty, especially in adolescents and the elderly, the impact of lowering the underreporting factor by $25 \%$ or $50 \%$ was also investigated.

In addition, scenario analyses were performed for several other vaccination strategies (Table $2 b$ ) including varying the age of the third booster dose between 5 and 35 years, using a combination schedule in which both adolescents (third booster dose) and adults (fourth booster dose) were vaccinated, and giving repeated booster doses every 10 years starting at age 10 years. 

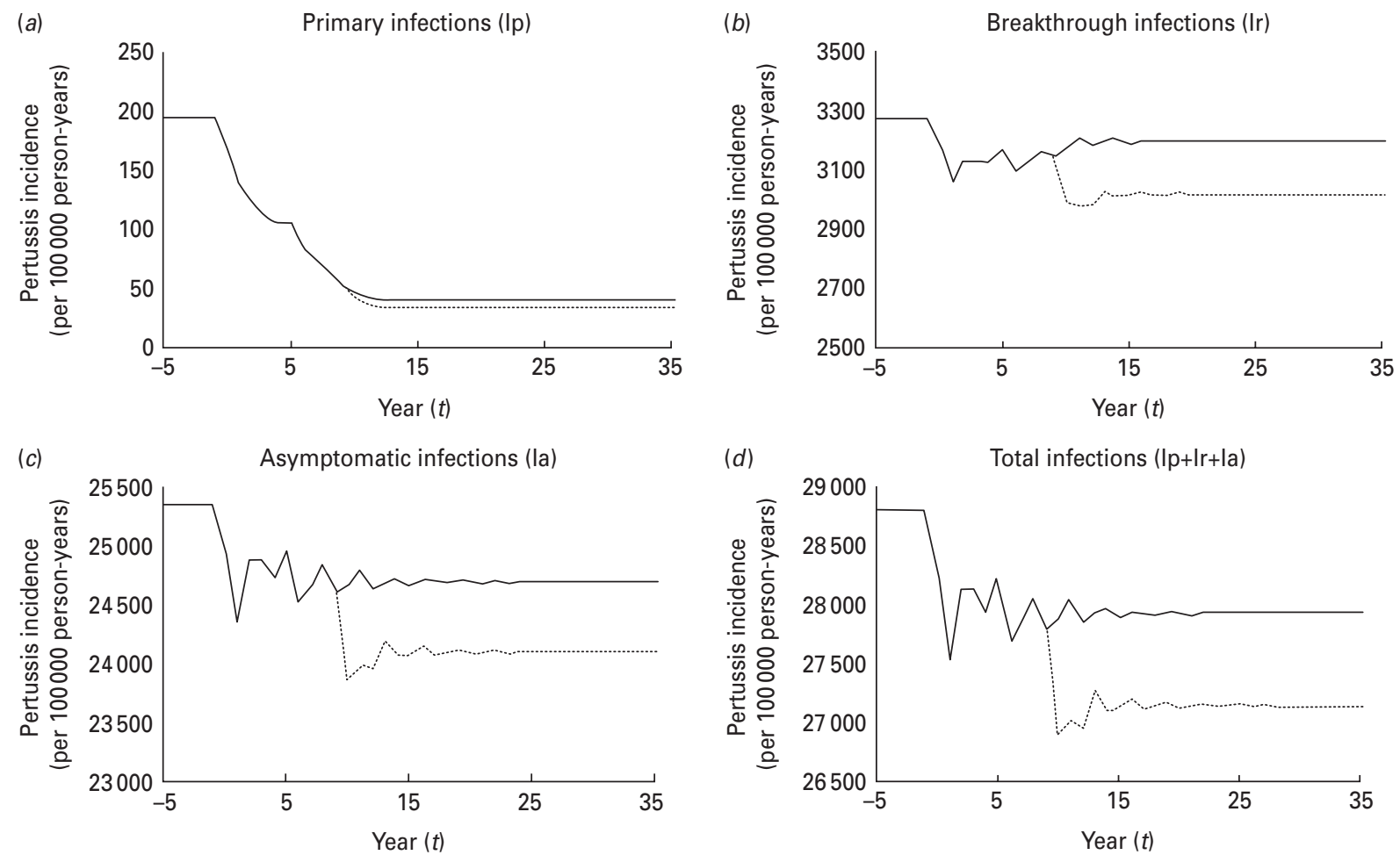

Fig. 2. Pertussis incidence/100 000 population per year applying base-case assumptions. The solid lines show the current situation after implementation of the booster dose at age 4 years (at $t=0$ ). The situation regarding adolescent vaccination after $t=10$ is represented by the dotted lines. Note that the y-axis does not start at 0 in all graphs.

\section{RESULTS}

\section{Base-case analysis}

The estimated impact of the implementation of two vaccination strategies, childhood boosters alone (at $t=0)$ and childhood boosters in combination with an adolescent booster vaccination (at $t=10$ ), on primary, breakthrough and asymptomatic pertussis infections is shown in Figure 2 (note that not all of the axes start at 0 ). The additional adolescent booster dose resulted in reduction of all types of pertussis infections with the relative decrease being most apparent for primary pertussis infections.

Although a decrease in overall infection was observed, the impact of the adolescent booster dose largely differed between age groups (Table 3 ). The largest absolute reduction and the largest relative reduction in infections were observed for children (6-12 years) and adolescents (13-19 years). Furthermore, although the total number of symptomatic infections declined as a result of the adolescent booster vaccination, the incidence in the older age groups increased, illustrating an age shift induced by the adolescent booster dose.

\section{Sensitivity analysis for base-case}

The impact of the different sensitivity analyses on the total number of infections is shown in Figure 3. Overall incidence of all infections decreased with increasing coverage of the adolescent booster vaccine at age 12 years (Fig. $3 a$ ). Furthermore, with increasing coverage, more primary and asymptomatic infections were averted in generally all age groups, while the shift in age of breakthrough infections became even more apparent.

Varying the transmission coefficients for the different types of infection resulted in a similar postvaccination endemic equilibrium (Fig. $3 b$ ). However, in the period between implementation of the booster dose and reaching the post-vaccination steadystate incidence (sometimes referred to as the posthoneymoon period), assumptions on the transmission coefficients, particularly for asymptomatic cases, had the most impact on overall pertussis incidence. For example, assuming that asymptomatic cases do not transmit infection resulted in stronger annual fluctuations in the incidence of breakthrough and asymptomatic infections, whereas assuming that asymptomatic 


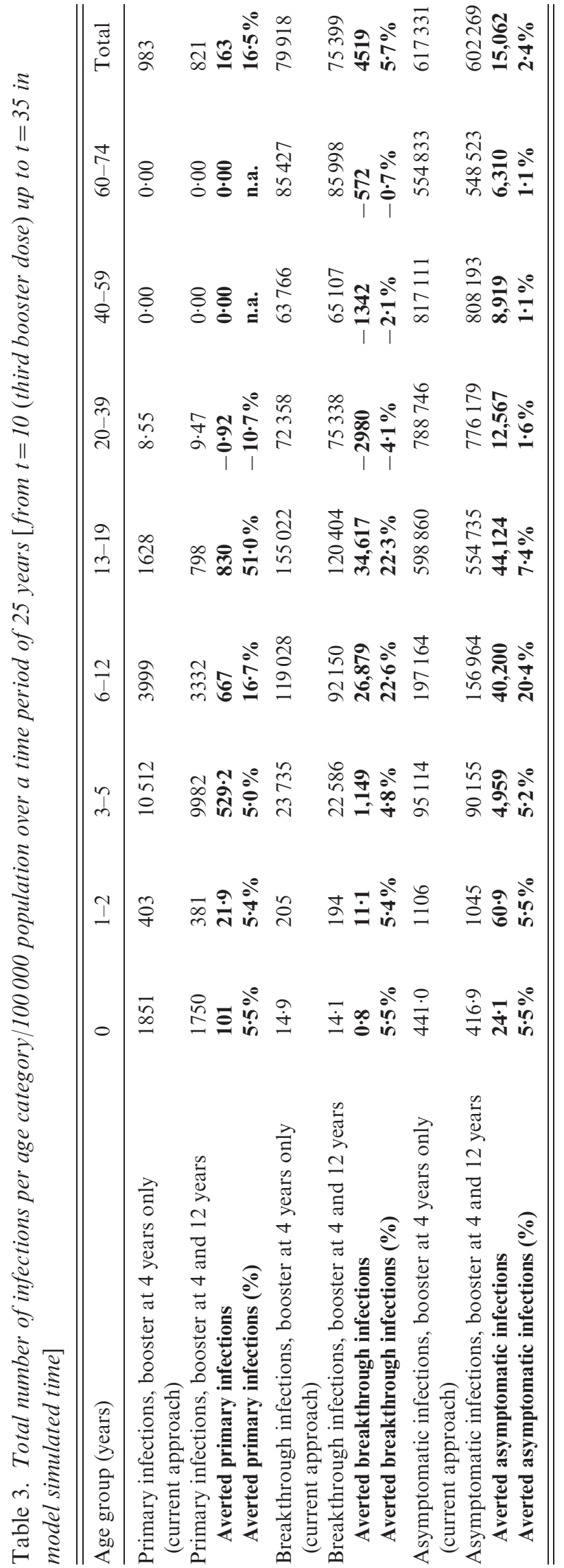

cases were more infectious than in the base-case analysis resulted in equilibrium being reached earlier.

Changing the duration of partial immunity after natural infection also did not have a considerable impact on the overall post-vaccination endemic equilibrium (Fig. 3 c). However, the incidence of breakthrough and asymptomatic cases differed substantially when the overall duration of protection was varied. The post-vaccination endemic equilibria for breakthrough and asymptomatic infections (per 100000) were 4400 and 22840 cases, respectively, assuming 6 years of partial protection, and 2360 and 24660 , respectively, assuming 14 years of protection. Nevertheless, the relative change in the number of cases was similar regardless of the duration of protection because, within the methodology used, the prevaccination pertussis incidence also differed proportionally to the post-vaccination endemic incidence. Shortening the infectious period for primary and breakthrough cases had almost no impact on the relative decrease of pertussis cases (Fig. $3 d$ ). In terms of absolute cases it was estimated that for the scenario when the infectious period for both primary and asymptomatic cases was set at 3 weeks, <0.34 cases/ 100000 population could be averted compared to the base case, where this was 0.46 cases $/ 100000$ population when the infectious period was reduced to 2 weeks.

Using the contact function of Mossong et al. [32] instead of the contact function of Wallinga et al. [31], resulted in the prevention of slightly more asymptomatic cases, although slightly less breakthrough infections were averted resulting in an overall similar endemic equilibrium (Fig. 3e). As expected, reducing the estimated underreporting factors resulted in lower total numbers of infections and in lower age-specific estimates of FOIs (Fig. 3f, Supplementary Fig. S1). Surprisingly, more pertussis cases were averted when the correction factor for underreporting was reduced by $25 \%$ compared to the base case, while slightly less cases were prevented when this factor was reduced by $50 \%$ compared to the base case.

\section{Scenario analyses}

The impact of varying the age of the third booster dose is shown in Figure 4. The optimal age of administering the third booster dose for the prevention of symptomatic cases was around 10 years, while the optimal age for the prevention of asymptomatic cases was around 12 years. The impact of adding a fourth 
(a)
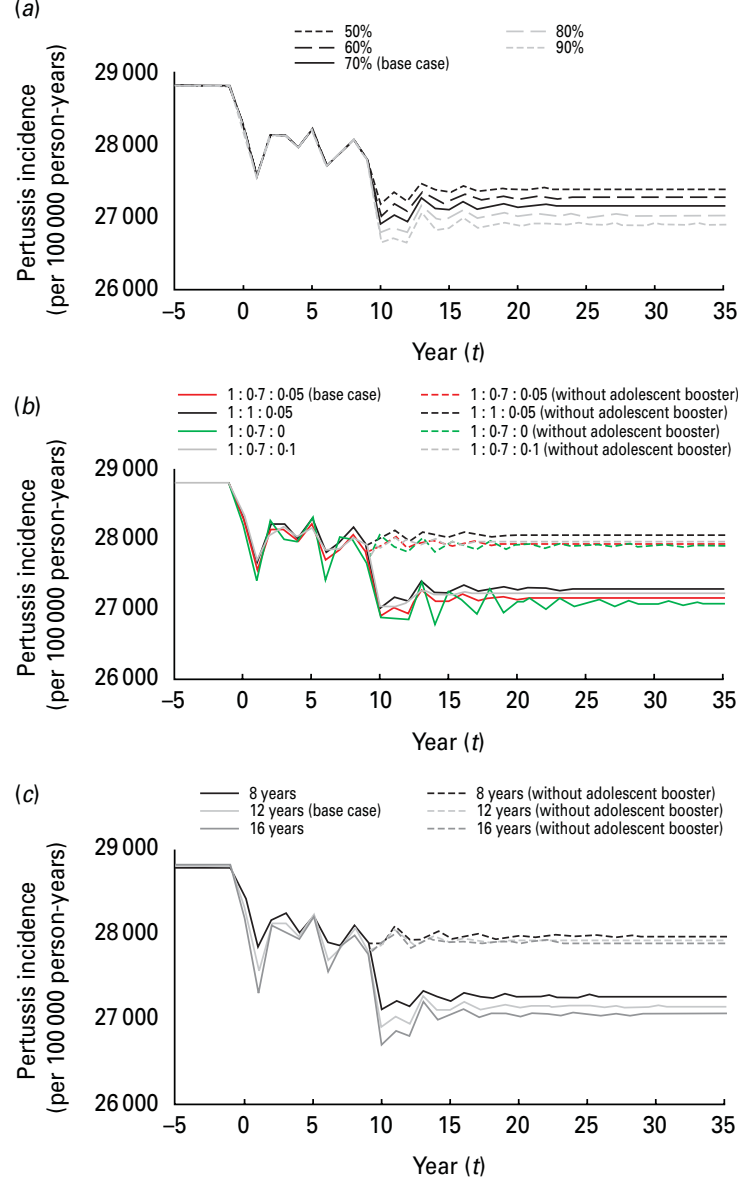
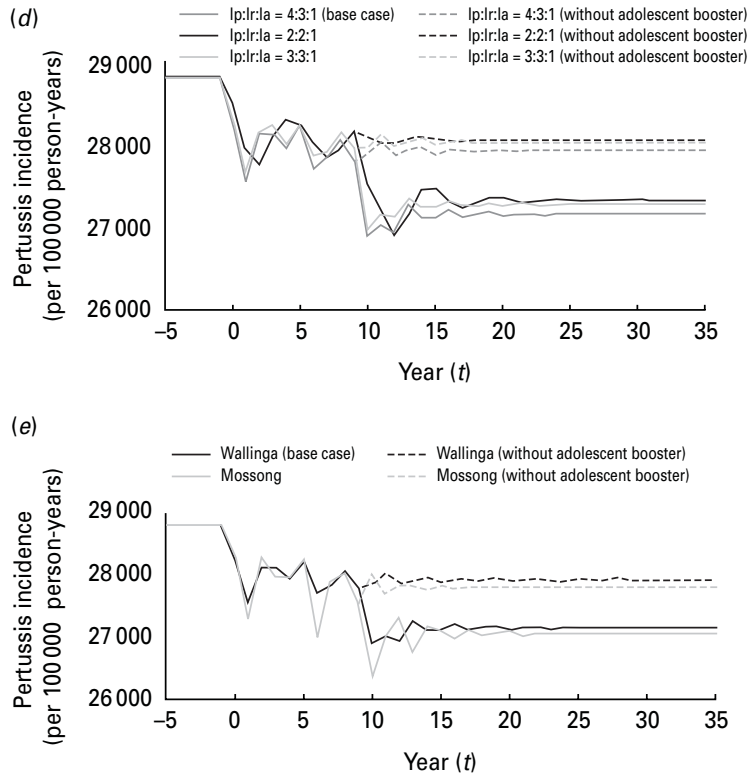

(f)

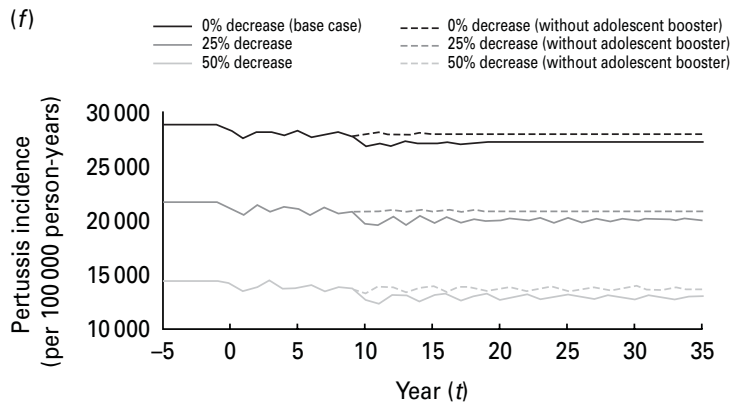

Fig. 3. Results of the sensitivity analyses of the base-case scenario (as described in the Methods section and Table 2). (a) The impact of varying the uptake of the booster dose between $50 \%$ and $90 \%(70 \%$ in the base-case) ; (b) the impact of using different transmission coefficients; $(c)$ the impact of assuming a different duration of protection after natural infection; $(d)$ the impact of reducing the infectious period; (e) the impact of applying the contact function is reported by Mossong et al. is shown; $(f)$ the impact of lowering the underreporting factor. Note that the y-axis does not start at 0 in any of the graphs.

booster was limited (data not shown). Nevertheless, we observed that the effective combination strategy for the prevention of symptomatic cases would be an additional childhood booster dose at age 10 years and an adult booster dose at age 20 years. However, the optimal strategy for the prevention of pertussis would require a booster dose every 10 years, starting at age 10 years (Fig. 5).

\section{DISCUSSION}

We have presented a realistic age-structured deterministic pertussis model, able to optimally use the scarce data on FOI and age-dependent fractions of symptomatic and notified cases. We have shown that, over a wide range of variations, an additional booster dose can reduce both the incidences of symptomatic and asymptomatic pertussis cases in the population.
Furthermore, we propose that the optimal timing for the third booster dose in The Netherlands is between the ages of 10 and 15 years and that the optimal vaccination strategy would be a booster dose every 10 years.

Notably, our results confirmed the epidemiological findings of a previous stochastic model in showing similar trends with respect to the prevention of pertussis cases and the induction of age shifts after the introduction of an additional adolescent booster dose [17].

The impacts of applying different assumptions on the overall disease burden were limited, although some assumptions (e.g. the duration of protection of natural protection) did change the ratio of asymptomatic and breakthrough cases. Surprisingly, the impact of an additional booster dose on the total reduction of pertussis cases was larger when the 
(a)

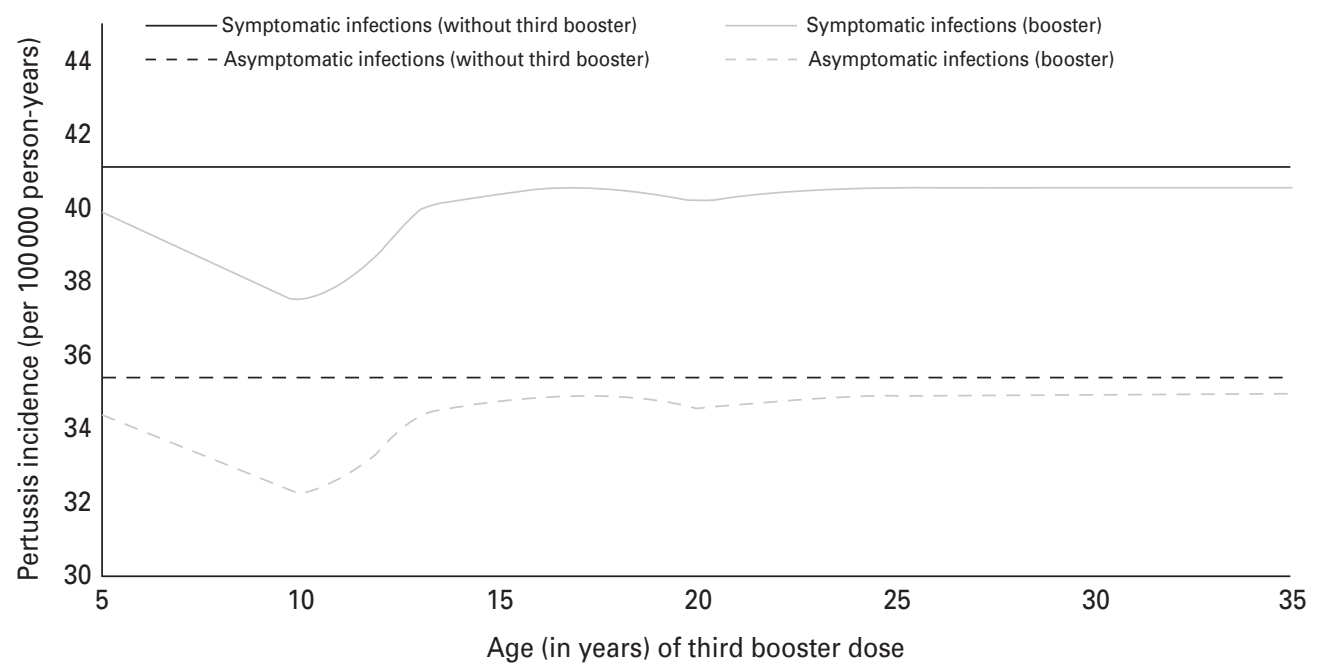

(b)

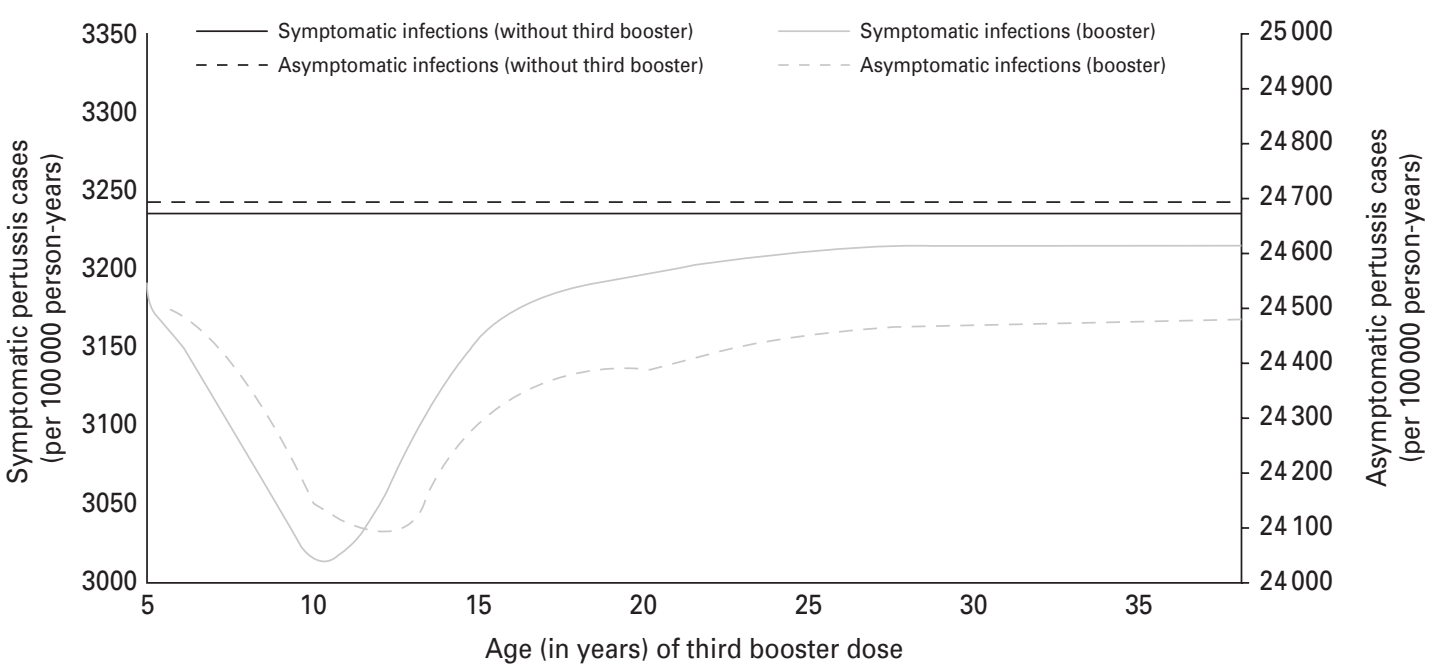

Fig. 4. $(a, b)$ Graphs showing the impact of varying the age of the third booster dose on the average number of symptomatic pertussis cases (solid lines) or asymptomatic pertussis cases (dashed lines) per age category/100 000 populations over a time period of 25 years after the introduction of a third booster dose $(t=10$ in model-simulated time). Horizontal lines represent the number of symptomatic cases (solid lines) or asymptomatic cases (dashed lines) without a third booster dose. (a) The impact on children aged $<3$ years; $(b)$ the impact on the total number of infections in the population.

underreporting factors was reduced by $25 \%$ from base-case level, while slightly fewer cases could be averted when the underreporting factors was reduced by $50 \%$ from base-case level. A possible explanation is that the infection pressure is so high in the basecase analysis that the impact of an additional booster dose will have only limited herd effects in the other unprotected individuals. More herd immunity effects may be observed if the FOI is lower (i.e. $25 \%$ or $50 \%$ lower underreporting), while on the other hand fewer cases can be prevented when the initial incidence is lower (50\% lower underreporting).

Several recent modelling studies used a dynamic model to estimate the effect of additional booster doses on the epidemiology of pertussis [14-19]. Most of these studies used estimates for FOI that were based on incidence data from England and Wales before the introduction of widespread vaccination. We used a method developed by van Boven et al. [21] to estimate specific Dutch FOIs which allowed waning of immunity and different types of infection to be taken into account. As a result, the FOI estimates of this study, corrected for age-specific underreporting and occurrence of asymptomatic infections, were consistently higher and of different shape than those previously reported [14-16, 18, 19].

Our model has a number of limitations. First, the method used to estimate the FOI assumed that the 


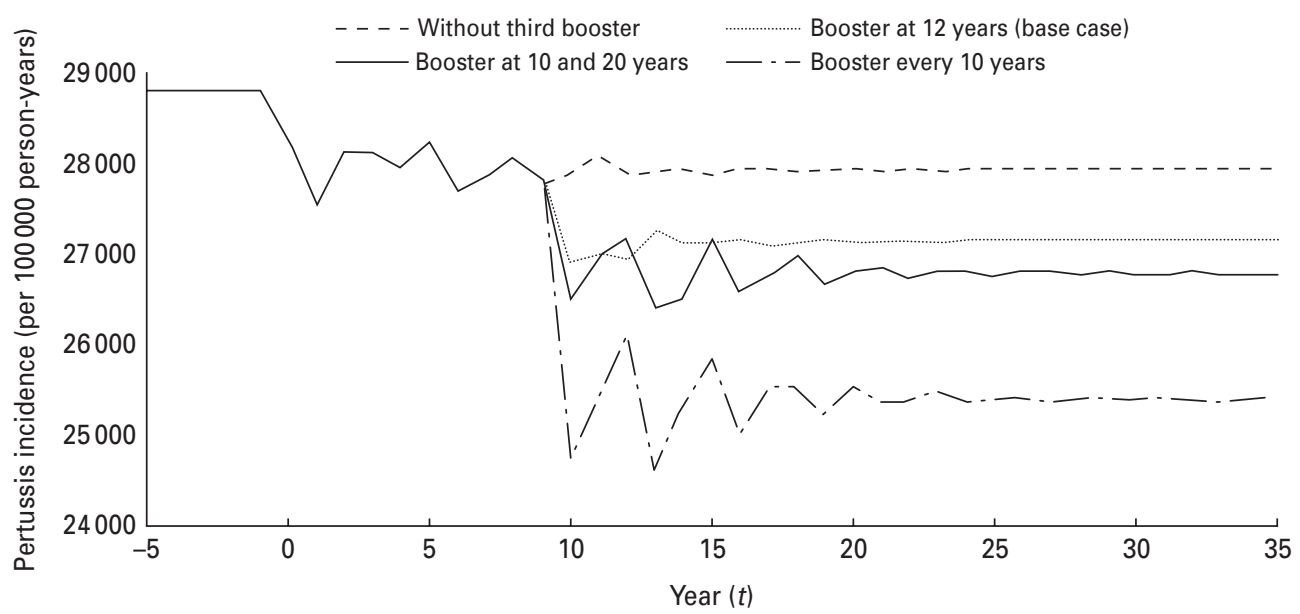

Fig. 5. Pertussis incidence/100 000 population per year showing the effect of a booster dose every 10 years (dashed-dotted line) compared to impact of the current situation (dashed line), the current situation combined with a potential single adolescent booster dose at age 12 years (dotted line, base-case analysis), and two additional booster doses at the ages of 10 and 20 years (solid line).

population is in endemic equilibrium during the period 1996-2000.

Although we are fully aware that this assumption does not reflect reality, we believe that it still represents the best approach currently available and that deviations from the steady state would not relevantly change the findings and conclusions. Second, the estimated incidence during this period and the underlying underreporting factors are uncertain. Our estimates of the incidence of infection are higher than estimated previously by de Melker et al. [29] for the period 1994-1996. This is because we used the incidence numbers of notified cases from 1996 to 2000 and increased these with age-specific underreporting factors from 1994 to 1996. As data from 1996 showed that the incidence was about six times higher compared to 7 years previously, our incidence estimates increased about sixfold [33]. As a result our model predicts that, on average, the entire population will be infected every 3 to 4 years, of which $88 \%$ will be asymptomatic. Nevertheless, as discussed above, when a lower overall incidence was applied, the number of averted cases increased when the underreporting rate was lowered to $25 \%$ while only a slight decrease in the number of averted case was observed when the underreporting rate was lowered to $50 \%$.

Similar to all previous modelling studies investigating the impact of additional pertussis booster doses [14, 16-19] we did not include maternal immunity. Indirect evidence from before the introduction of widespread vaccination indicated that maternal antibodies provided some protection against mortality during the first month of life [34]. However, surveillance data after the introduction of widespread pertussis vaccination no longer show this relation between maternal antibodies and protection against pertussis [34]. Furthermore, as there is no serological correlate of protection for pertussis it is not possible to estimate the proportion of infants born with a protective level of maternal antibodies [34]. Therefore, we decided not to include maternal immunity in our model.

We used equally sized cohorts assuming a type 1 mortality, rather than the actual age distribution of The Netherlands. It has been argued in the literature that this approximation represents a valid approach for developed countries [35].

Last, it should be noted that an economic evaluation of our analyses in order to judge the attractiveness of the different strategies from the costeffectiveness perspective is warranted. The most costeffective strategy may certainly differ from the most optimal vaccination strategy (i.e. a booster dose every 10 years), in terms of primary and/or total cases averted. Thus, an economic evaluation, using the epidemiological results reported here, will be the next step in the evaluation of potential additional pertussis vaccination strategies in the Dutch context.

In conclusion, we designed an age-structured deterministic pertussis model with rapid simulation runs, which was used to explore the impact of various parameter assumptions and pertussis booster vaccination strategies on pertussis epidemiology. We showed that the optimum age of an additional booster 
dose is between ages 10 and 15 years, while the optimal vaccination strategy is a booster dose every 10 years. A sensible strategy representing a compromise between these two approaches may be booster vaccinations at 10 and 20 years.

\section{NOTE}

Supplementary material accompanies this paper on the Journal's website (http://journals.cambridge.org/ hyg).

\section{ACKNOWLEDGEMENTS}

We thank the reviewers for their helpful suggestions.

\section{DECLARATION OF INTEREST}

This research was supported by an unrestricted grant from GSK Bio (Wavre, Belgium). R.dV. is currently an employee of Roche (Woerden, The Netherlands). M.J.P. and M.H.R. have received travel grants and honoraria from various vaccine producers.

\section{REFERENCES}

1. Celentano LP, et al. Resurgence of pertussis in Europe. Pediatric Infectious Disease Journal 2005; 24: 761-765.

2. Ntezayabo B, de Serres G, Duval B. Pertussis resurgence in Canada largely caused by a cohort effect. Pediatric Infectious Disease Journal 2003; 22 : 22-27.

3. Quinn HE, McIntyre PB. Pertussis epidemiology in Australia over the decade 1995-2005 - trends by region and age group. Communicable Diseases Intelligence 2007; 31 : 205-215.

4. de Melker HE, et al. Pertussis in The Netherlands: an outbreak despite high levels of immunization with whole-cell vaccine. Emerging Infectious Diseases 1997; 3: $175-178$

5. de Greeff SC, et al. Impact of acellular pertussis preschool booster vaccination on disease burden of pertussis in The Netherlands. Pediatric Infectious Disease Journal 2008; 27 : 218-223.

6. Guris D, et al. Changing epidemiology of pertussis in the United States: increasing reported incidence among adolescents and adults, 1990-1996. Clinical Infectious Diseases 1999; 28: 1230-1237.

7. Skowronski DM, et al. The changing age and seasonal profile of pertussis in Canada. Journal of Infectious Diseases 2002; 185: 1448-1453.

8. Bisgard KM, et al. Infant pertussis: who was the source? Pediatric Infectious Disease Journal 2004; 23 : 985-989.

9. Elliott E, et al. National study of infants hospitalized with pertussis in the acellular vaccine era. Pediatric Infectious Disease Journal 2004; 23 : 246-252.
10. Wendelboe AM, et al. Transmission of Bordetella pertussis to young infants. Pediatric Infectious Disease Journal 2007; 26: 293-299.

11. Halperin SA. Canadian experience with implementation of an acellular pertussis vaccine booster-dose program in adolescents: implications for the United States. Pediatric Infectious Disease Journal 2005; 24: S141-S146.

12. Tan T, Trindade E, Skowronski D. Epidemiology of pertussis. Pediatric Infectious Disease Journal 2005; 24 : $\mathrm{S} 10-\mathrm{S} 18$

13. de Greeff SC, et al. Pertussis disease burden in the household: how to protect young infants. Clinical Infectious Diseases 2010; 50: 1339-1345.

14. Coudeville L, van Rie A, Andre P. Adult pertussis vaccination strategies and their impact on pertussis in the United States: evaluation of routine and targeted (cocoon) strategies. Epidemiology and Infection 2008; 136: 604-620.

15. van Boven M, et al. A model based evaluation of the 1996-7 pertussis epidemic in The Netherlands. Epidemiology and Infection 2001; 127: 73-85.

16. Coudeville $\mathbf{L}$, et al. Adult vaccination strategies for the control of pertussis in the United States: an economic evaluation including the dynamic population effects. PLoS One 2009; 4: e6284.

17. de Vries R, et al. Cost-effectiveness of adolescent pertussis vaccination for The Netherlands: using an individual-based dynamic model. PLoS One 2010; 5: e13392.

18. Hethcote HW, Horby P, McIntyre P. Using computer simulations to compare pertussis vaccination strategies in Australia. Vaccine 2004; 22: 2181-2191.

19. Van Rie A, Hethcote HW. Adolescent and adult pertussis vaccination: computer simulations of five new strategies. Vaccine 2004; 22: 3154-3165.

20. Wendelboe AM, et al. Duration of immunity against pertussis after natural infection or vaccination. Pediatric Infectious Disease Journal 2005; 24: S58-S61.

21. van Boven M, et al. Waning immunity and sub-clinical infection in an epidemic model: implications for pertussis in The Netherlands. Mathematical Biosciences 2000; 164: 161-182.

22. Farrington $\mathbf{C P}$. The measurement and interpretation of age-specific vaccine efficacy. International Journal of Epidemiology 1992; 21: 1014-1020.

23. Ramsay ME, Farrington CP, Miller E. Age-specific efficacy of pertussis vaccine during epidemic and non-epidemic periods. Epidemiology and Infection 1993; 111: 41-48.

24. Jefferson T, Rudin M, DiPietrantonj C. Systematic review of the effects of pertussis vaccines in children. Vaccine 2003; 21 : 2003-2014.

25. Zhang $\mathbf{L}$, et al. Acellular vaccines for preventing whooping cough in children. Cochrane Database of Systematic Reviews 2009. Issue 4. Art No.: CD001478.

26. Casey JR, Pichichero ME. Acellular pertussis vaccine safety and efficacy in children, adolescents and adults. Drugs 2005; 65: 1367-1389. 
27. Frampton JE, Keam SJ. Reduced-antigen, combined diphtheria-tetanus-acellular pertussis vaccine, adsorbed (Boostrix) US formulation: use as a single-dose booster immunization in adolescents aged 10-18 years. Paediatric Drugs 2006; 8: 189-195.

28. Ward JI, et al. Efficacy of an acellular pertussis vaccine among adolescents and adults. New England Journal of Medicine 2005; 353: 1555-1563.

29. de Melker HE, et al. The incidence of Bordetella pertussis infections estimated in the population from a combination of serological surveys. Journal of Infection 2006; 53: 106-113.

30. De Greeff SC, et al. Pertussis in the Netherlands, 2001-2002. RIVM report 128507010/2003.

31. Wallinga J, Teunis $\mathbf{P}$, Kretzschmar M. Using data on social contacts to estimate age-specific transmission parameters for respiratory-spread infectious agents. American Journal of Epidemiology 2006; 164: 936944.

32. Mossong $\mathbf{J}$, et al. Social contacts and mixing patterns relevant to the spread of infectious diseases. PLoS Medicine 2008; 5: e74.

33. de Greeff SC, et al. Effect of vaccination against pertussis on the incidence of pertussis in The Netherlands, 1996-2003 [in Dutch]. Nederlands Tijdschrift Voor Geneeskunde 2005; 149: 937-943.

34. van Rie A, Wendelboe AM, Englund JA. Role of maternal pertussis antibodies in infants. Pediatric Infectious Disease Journal 2005; 24: S62-S65.

35. Anderson RM, May RM. Infectious Diseases of Humans. Oxford: Oxford University Press, 1991. 